\title{
La sécurité de l'alimentation en eau potable d'une grande agglomération - Nouvelle approche méthodologique
}

\author{
M. Bué \\ Communauté Urbaine de Lyon. Direction de l'Eau
}

\section{Le contexte de l'étude}

L'alimentation en eau potable de la Communauté Urbaine de Lyon concerne une population de plus d'un million d'habitants, répartie sur plus de $500 \mathrm{~km}^{2}$, à partir de deux sites de production principaux et d'une dizaine de petits captages $\left(320000 \mathrm{~m}^{3} /\right.$ jour en moyenne, $450000 \mathrm{~m}^{3} /$ jour en pointe). Elle met en jeu $3500 \mathrm{~km}$ de conduites ainsi qu'une soixantaine de stations-relais et réservoirs.

Améliorer la sécurité de son système d'alimentation est, pour la Communauté Urbaine comme pour de nombreuses collectivités, l'objectif de la plupart des investissements depuis une dizaine d'années. Parmi les investissements majeurs, on peut citer la création d'une diversification de la ressource, le développement des interconnexions entre réseaux principaux, le doublement de feeders alimentant certaines zones de l'agglomération. D'autre part, au-delà de ces grands équipements, l'amélioration de la sécurité de l'alimentation est un critère déterminant des politiques de renouvellement des installations.

Cet effort de plusieurs années ayant fait incontestablement progresser la sécurité de l'alimentation en eau de l'agglomération. Il a paru toutefois utile aux responsables de la Communauté Urbaine, tant pour apprécier les progrès accomplis que pour préciser les politiques techniques dans le domaine de l'eau et orienter d'éventuels futurs investissements, de procéder à une analyse méthodique de la sûreté de l'alimentation en eau.

Une telle approche méthodique de la sécurité répondait également au souci exprimé par l'Agence de l'Eau Rhône Méditerranée Corse de disposer d'une réflexion globale et prospective sur l'alimentation en eau potable de la plus grande agglomération du Bassin.
1. Objectifs, déroulement et méthode de l'étude de sûreté

\subsection{Objectifs de l'étude de sûreté}

L'étude a dû répondre aux objectifs principaux suivants : - établir le diagnostic de la sécurité du système d'alimentation en eau potable de l'agglomération,

- identifier les maillons faibles du système, au regard de l'ensemble des risques auquel il est confronté ; élaborer une grille permettant de caractériser ces maillons faibles (en fonction de la nature des risques associès, de leur probabilité et de la gravité de leurs conséquences...) et de hiérarchiser leur importance par rapport à la sûreté du système, - repérer les solutions (procédures d'exploitation, investissements complémentaires...) permettant de renforcer le niveau de sécurité des maillons faibles préalablement identifiés, puis comparer ces solutions au regard des gains de sécurité qu'elles peuvent apporter.

\subsection{Déroulement de l'étude}

Cette étude a été suivie par un Comité de Pilotage, présidé par Monsieur F. BLanc, Vice-Président de la Communauté Urbaine de Lyon, auquel a participé l'Agence de l'Eau Rhône Méditerranée Corse.

Les orientations générales de l'étude ont été établies par l'Agence de l'Eau, dans une note préliminaire qui a servi de fil conducteur à la Compagnie Générale des Eaux et à la Société Générale de Maintenance (spécialiste de la fiabilité industrielle) pour la proposition de la méthode de travail. 
Cette méthode a reposé sur l'emploi d'outils méthodologiques (Analyse Fonctionnelle, Analyse Préliminaire de Risques) qui ont guidé le travail de plusieurs groupes réunissant les partenaires techniciens (Générale des Eaux, Communauté Urbaine de Lyon, Agence de l'Eau), animés par des ingénieurs de la Générale de Maintenance.

Pour valider la méthode proposée, le travail a d'abord porté sur un sous-ensemble du système d'alimentation en eau potable de l'agglomération (la ressource et le réseau de distribution dit "Haut Service») avant d'être étendu à l'ensemble des composants du réseau.

La méthodologie employée a comporté trois étapes :

Etape 1: Analyse de l'existant: Cette étape consiste à porter, pour la situation actuelle, une réflexion sur l'organisation du système d'alimentation en eau potable, en spécifiant en particulier les fonctions à remplir par les différents composants du système et les conditions à remplir pour que ces fonctions soient correctement assurées. Elle se compose :

- d'une analyse fonctionnelle du réseau qui décrit la structure du système (son organisation, sa décomposition en sous-systèmes, ensembles, composants élémentaires et les liaisons entre les différents composants du système), précise les différentes fonctions assurées par les composants du système et des liens entre ces fonctions ;

- de l'établissement d'un document (le « découpage organique " du système) dans lequel sont reprises toutes les informations caractéristiques du réseau (géographiques, quantitatives...);

- de l'analyse des plans de secours actuels.

Le résultat de cette étape se présente sous la forme de tableaux des fonctions que doit remplir le système, dans lequel ressortent les couples formés par les fonctions (hiérarchisées) et les éléments concernés par ces fonctions.

Etape 2: Etude de sécurité : Cette étape repose sur l'application d'une méthode appelée Analyse Préliminaire de Risques (APR), dont l'objectif est :

- d'identifier les risques pesant sur le système et leurs causes. Il s'agit tout d'abord, sur la base de l'analyse effectuée à l'étape 1 , et en tenant compte des pertes de fonction qu'une défaillance peut engendrer, d'inventorier les composants du système auxquels on peut associer un risque particulier (ces composants sont alors repérés comme " entités dangereuses »). Il s'agit ensuite de décliner les conditions qui doivent être réunies pour rendre ces entités véritablement dangereuses, l'accent étant mis ici sur l'interaction entre entité et facteur déclenchant (on identifie ainsi les " situations dangereuses " et les " accidents potentiels " susceptibles de provoquer la perte d'une fonction). On détermine ainsi l'ensemble des "situations à risques " susceptible d'affecter l'alimentation en eau potable de tout ou partie de l'agglomération ;

- d'évaluer l'importance de ces « situations à risques ", en les caractérisant par une variable " 1 "indice de criticité ", qui intègre la probabilité d'apparition du risque, la gravité de ses conséquences et la détectabilité des situations dangereuses ;
- de rappeler ou de définir les actions correctives qui doivent permettre d'éliminer ou de maîtriser les "situations à risques ". Le principe de quantification (indice de criticité) permettra d'apprécier l'apport en sécurité des mesures réalisées ou envisagées.

Cette méthode a été employée en groupes de travail animés par la Générale de Maintenance réunissant des exploitants, des responsables communautaires et des représentants de l'Agence de Bassin.

Elle a permis de formaliser l'expertise des différents techniciens, dans la recherche du maximum d'exhaustivité et d'objectivité quant aux risques menaçant le système et à leurs conséquences.

Etape 3 : Classification des risques et recommandations Stratégies de sécurisation: Les risques et les mesures correctives peuvent alors être classifiés en utilisant les critères suivants :

— pour les situations à risques, la valeur de l'indice de criticité actuel.

- pour les mesures correctives, la valeur du gain potentiel en sécurité constitué par la différence des indices de criticité avant et après la mise en cuvre de la mesure.

Ce calcul est ensuite apprécié en valeur relative :

- à un risque donné, on peut attribuer une proportion du risque total pesant sur le système d'alimentation en eau potable.

- à une mesure corrective donnée, on peut affecter une proportion du gain de sécurité total envisageable pour le système.

Les résultats de ces calculs permettent de mettre en perspective les différents risques et les mesures correctives identifiées et de guider les responsables dans l'élaboration des politiques d'amélioration de la sécurité

\section{Evaluation du risque et de l'apport des mesures}

\subsection{L'appréciation aussi objective que possible de l'impor- tance de chacune des " situations à risques " examinées en groupe de travail est un élément essentiel de la méthode employée, dont les principes doivent être établis avant la mise en auvre de l'Analyse Préliminaire de Risques}

Il a donc fallu en premier lieu élaborer les critères d'appréciation des différentes situations à risque. Les critères finalement retenus sont les suivants :

- la probabilité d’apparition du risque.

- la gravité des conséquences d'un accident potentiel.

- la possibilité de détection préalable de la situation à risque,

Ces critères ont par ailleurs fait l'objet d'une pondération, traduisant les objectifs politiques de la Communauté Urbaine de Lyon. 


\section{SÉCURITÉ DE L'ALIMENTATION EN EAU POTABLE}

L'application des différents critères a conduit à définir un indice de criticité (produit pondéré des notes obtenues pour chacun des critères).

Cette méthode a donc été employée pour apprécier la criticité des différentes situations à risques, et ce :

- dans la situation actuelle des installations (en tenant compte des procédures d'équipements déjà présents);

- dans une situation future, où auraient été mis en place les équipements ou procédures repérés comme susceptibles d'améliorer la sécurité.

La différence des deux indices (criticité actuelle - criticité future) permet de quantifier l'apport "en sécurité " de chaque mesure de sécurisation.

\subsection{Présentation des différents critères}

\section{La probabilité d'apparition du risque :}

La gravité: L'étude a fait ressortir que l'on pouvait tenter d'apprécier la gravité d'un accident potentiel en fonction :

a) de ses effets sur le niveau de service assuré par le système d'alimentation en eau potable (en quelque sorte l'intensité du dommage), que ce soit au niveau de la quantité d'eau distribuable ou de sa qualité.

b) de l'importance des populations concernées et de la nature des usages (prioritaires ou non), en quelque sorte l'étendue du dommage.

c) de l'autonomie ou de la « résilience » du réseau concerné (sa capacité structurelle à faire face à l'accident), et de la façon dont celle-ci est affectée par l'accident ;

d) des effets induits par l'accident, sur la sécurité publique (biens et personnes), sur la sécurité des personnels intervenant, sur les coûts directs de réparation de l'accident.

La détectabilité : La possibilité de détection préalable de l'événement causant une situation dangereuse concourt bien sûr à qualifier son importance. Le risque affectera d'autant moins le système qu'il existera des signes avantcoureurs permettant de prendre des mesures préventives, et d'accroître l'autonomie des réseaux.

\section{Résultats de l'étude}

\subsection{Evaluation des risques}

A titre indicatif, nous rappelons ci-dessous les principaux facteurs de risque pris en compte dans l'analyse.

Pour la ressource :

- les facteurs météorologiques (température très élevée ou très basse, foudre) ;

- les facteurs hydrologiques (étiage ou crue);

- les facteurs concernant la qualité de l'eau brute ou de nappe (turbidité liée aux vidanges de barrages, pollutions...);

- les perturbations de l'alimentation électrique ;
Pour l'ensemble des organes du réseau (usines principales, stations relais, conduites, vannes, réservoirs...) :

- les risques liés au milieu extérieur (dommages de travaux publics, glissement de terrain, incendie, intrusion, malveillance...);

- les perturbations de l'alimentation électrique ;

- les dysfonctionnements des équipements de contrôle et de transmissions des informations (capteurs, actionneurs, défaillance logique ou électrique des automates...);

- les pannes d'équipements électromécaniques ;

- les retours d'eau polluée ;

- les fausses manœuvres.

L'Analyse Préliminaire de Risques a conduit à examiner l'impact de chacun de ces risques sur les différents composants des réseaux, ce qui a permis d'identifier plus d'une centaine de "situations à risques", dont la criticité a été appréciée selon les critères rappelés précédemment.

Par sommation, il est ainsi possible d'évaluer la criticité actuelle des différentes familles de risques, comme le montre la figure 1 .

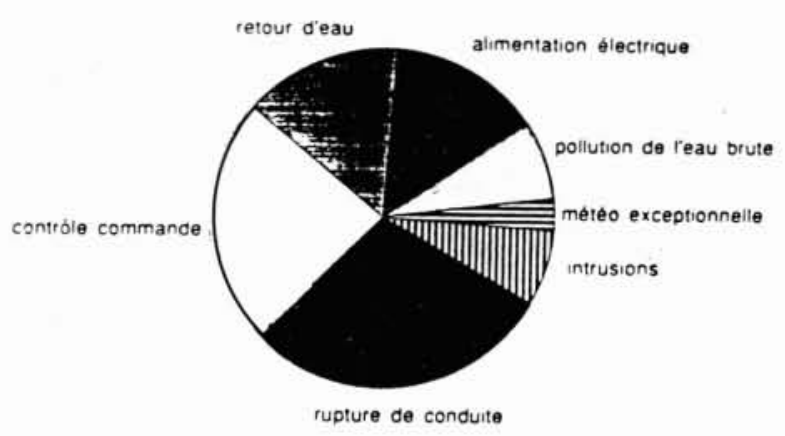

1. Répartition des risques par famille.

Cette analyse fait donc ressortir la part prépondérante des risques "décentralisés" par rapport aux risques centralisés (tels ceux qui affectent la ressource).

Ce résultat apparemment paradoxal est en fait la conséquence :

- des investissements déjà nombreux engagés par la Communauté Urbaine de Lyon sur la ressource ;

- de la très grande diffusion d'un certain nombre de risques, qui rendait délicate leur appréciation et leur réduction, et qui est fortement valorisée dans une approche exhaustive du type de celle qui a été développée.

Le poids des risques décentralisés, et notamment de ceux qui affectent l'interface avec l'abonné constitue indéniablement un résultat important de l'étude, qui doit conduire à certains réajustements de priorités. 


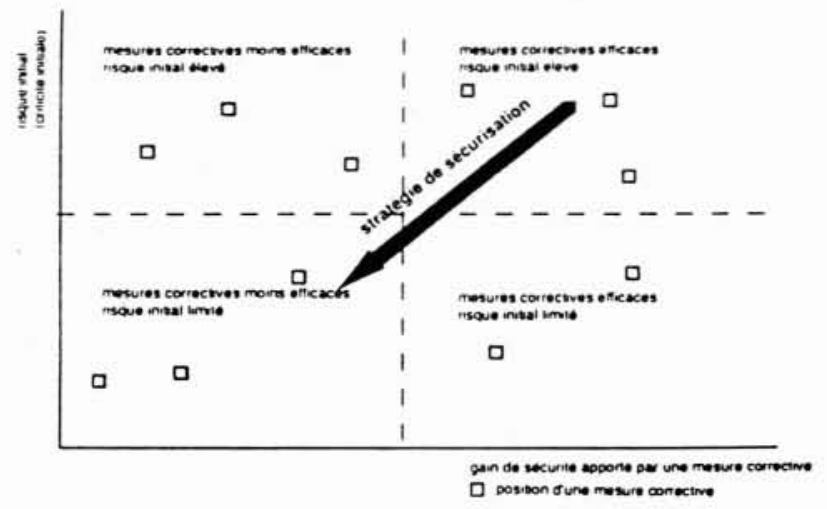

2. Diagramme criticité - gain de sécurité.

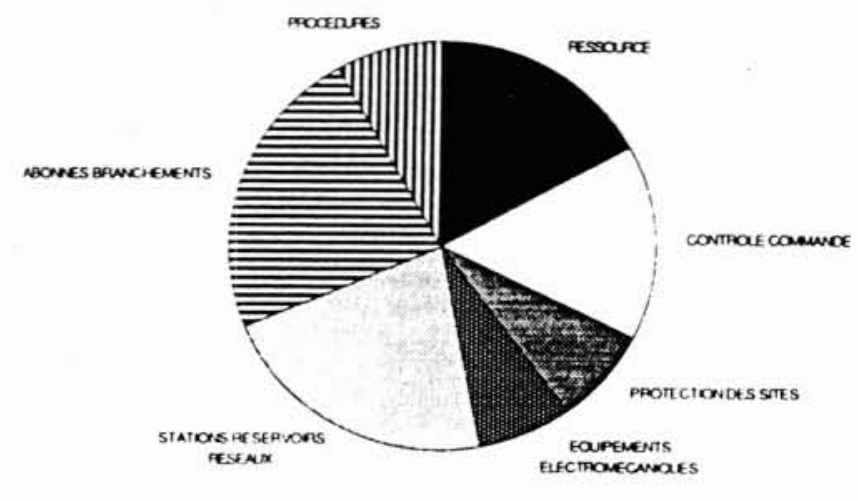

3. Répartition des mesures correctives par famille.

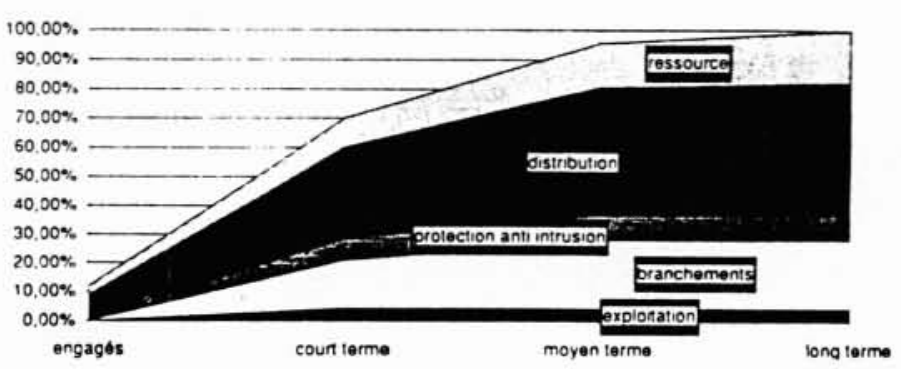

4. Evolution des gains de sécurité par politique.

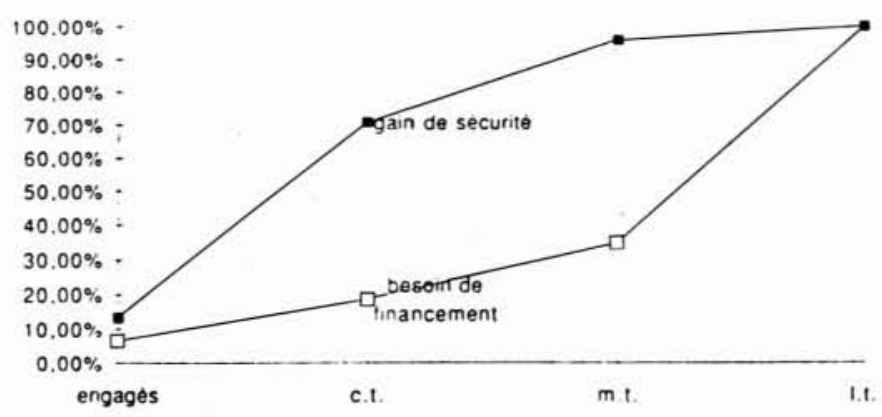

5. Evolution des gains de sécurité par politique. 


\subsection{Mesures correctives}

\section{Evaluation des mesures correctives}

Les mesures correctives recensées en groupe de travail, au cours de l'Analyse Préliminaire des Risques, ont été caractérisées par la réduction de l'indice de criticité qu'apporte la mesure corrective à la situation à risques.

Elles ont alors été hiérarchisées avec plusieurs outils, dont : le diagramme "Criticité - Gain de Sécurité ", dans lequel on situe la position des différentes mesures correctives sur un diagramme à deux axes, où figurent :

- en abscisse, le "Gain de Sécurité " (toujours défini comme la différence des indices de criticité avant et après mise en œuvre de la mesure corrective);

- en ordonnée, l'indice de criticité initial de la situation à risques («le risque initial »).

Sur ce diagramme :

- les mesures correctives attachées aux risques les plus sensibles figurent en partie supérieure;

- les mesures correctives les plus efficaces sont situées dans la partie droite.

Une politique de sécurisation s'attachera donc à mettre en œuvre en priorité les mesures correctives situées en haut à droite, en convergeant progressivement vers l'origine du tableau (voir fig. 2) et en intégrant bien sûr d'autres critères, tels les aspects financiers.

\section{Les mesures correctives recensées}

Chaque mesure corrective a donné lieu à l'établissement d'une fiche de présentation, qui la décrit sommairement et rappelle les risques auxquels elle peut servir de parade. Les mesures correctives ont été regroupées par famille, dont l'intérêt en termes de gain de sécurité a été apprécié (voir fig. 3). Cette analyse, croisée avec l'utilisation du diagramme "Criticité - Gain de Sécurité », a fait ressortir que la sécurisation de l'alimentation en eau potable de l'agglomération devait prendre en compte cinq grandes dimensions, qui constituent autant de politiques à mettre en œuvre.

Les politiques de sécurisation sont les suivantes:

- une politique de sécurisation de la ressource (mettant en œuvre $18 \%$ des gains de sécurité potentiels à travers 15 mesures), allant de la réalisation d'aménagements mineurs (procédures, outils informatiques de gestion de la nappe) à la réalisation d'investissements plus lourds (réalimentation de la nappe, extension de filière de traitement, voire diversification complémentaire de la ressource);

- une politique de sécurisation de la distribution, représentant $45 \%$ des gains de sécurité, et recouvrant : un volet portant sur le système de contrôle commande (18 mesures identifiées, $16 \%$ des gains de sécurité) ; un volet de renforcement de l'autonomie et des moyens de secours des différents réseaux (mesures portant sur les stations-relais, les réservoirs et les conduites de transport - 29 mesures identifiées, $22 \%$ des gains de sécurité) : un volet portant sur les équipements électromécaniques (4 mesures, $7 \%$ des gains de sécurité);

- une politique de protection des ouvrages contre les intrusions (comptant pour $8 \%$ des gains de sécurité);
— une politique de sécurisation des points de livraison (principalement la protection anti-retour des réseaux, représentant $24 \%$ des gains de sécurité);

— une politique de sécurisation de l'exploitation (5\% des gains identifiés).

A part pour certaines mesures dont la mise en place a pû être immédiate, ces politiques ont fait l'objet d'une programmation, intégrant à la fois les aspects sécuritifs et financiers, et définissant trois termes pour la mise en œuvre des différentes mesures : le court terme ( 5 ans); le moyen terme (10 ans); le long terme (20 ans).

La figure 4 présente le développement des politiques, en précisant, pour chacun des termes, les gains de sécurité générés par les différentes politiques. La figure 5 met en regard les besoins de financement correspondants. On vérifie ainsi que les mesures sont engagées à « coût d'obtention de la sécurité " croissant.

\section{Conclusions}

La méthode mise au point pour cette étude de la sûreté de l'alimentation en eau potable de la Communauté Urbaine de Lyon s'est révélée fructueuse à plusieurs titres :

- les outils d'évaluation des risques et des mesures correctives ont fourni des indications précieuses pour l'élaboration des politiques de sécurisation. La méthode a en effet permis : de comparer les effets à attendre des différentes politiques, et d'arbitrer sur les priorités ; de hiérarchiser les différentes mesures au sein d'une même famille (ex. : choix des réservoirs à accroître en priorité); d'intégrer toutes les dimensions concourant à l'amélioration de la sécurité, et notamment celle des procédures d'exploitation, qui est souvent laissé de côté dans les approches traditionnelles; - cette étude permet de disposer d'un outil méthodologique utilisable pour réajuster périodiquement les différentes politiques techniques;

- la méthode employée s'attache à faire ressortir l'ensemble des risques résiduels susceptibles de mettre en cause les fonctions du système ou d'une partie du système.

Elle permet de formaliser l'expertise des techniciens avec souplesse (dans le choix des fonctions retenues - d'abord générales, puis focalisées sur des éléments critiques) et exhaustivité.

- l'association des exploitants et des partenaires extérieurs (Direction de l'Eau, Agence de l'Eau) dans les groupes de travail et la méthode d'APR employée sont des facteurs très positifs pour la sensibilisation des acteurs aux enjeux de la sécurité. Certaines mesures ont été engagées immédiatement suite à leur mise en évidence ;

- l'étude fait ressortir l'intérêt de prolongements sur des domaines plus spécifiques (étude de fiabilité de type AMDEC pour certains équipements "critiques ", développement des politiques de maintenance préventive...); - les enseignements tirés de cette expérience permettent d'envisager sa transposabilité à d'autres sites ou d'autres types de réseaux dans de bonnes conditions. 